\title{
Coulisses
}

Revue de théâtre

19 | Hiver 1999

Varia

\section{Théâtre latino-américain contemporain (1940-1990)}

Textes réunis et présentés par Osvaldo Obregón

\section{Osvaldo Obregón}

Traducteur : Albert Bensoussan

\section{(2)enEdition}

\section{Journals}

Édition électronique

URL : https://journals.openedition.org/coulisses/5733

DOI : $10.4000 /$ coulisses.5733

ISSN : 2546-9460

\section{Éditeur}

Presses universitaires de Franche-Comté

\section{Édition imprimée}

Date de publication : 1 janvier 1999

Pagination : 63-66

ISBN : 2-913322-09-3

ISSN : 1150-594X

\section{Référence électronique}

Osvaldo Obregón, «Théâtre latino-américain contemporain (1940-1990) », Coulisses [En ligne], 19| Hiver 1999, mis en ligne le 18 octobre 2019, consulté le 04 janvier 2022. URL : http://

journals.openedition.org/coulisses/5733; DOI : https://doi.org/10.4000/coulisses.5733

Ce document a été généré automatiquement le 4 janvier 2022

Coulisses 


\section{Théâtre latino-américain contemporain (1940-1990)}

Textes réunis et présentés par Osvaldo Obregón ${ }^{1}$

\section{Osvaldo Obregón}

Traduction : Albert Bensoussan

1 Depuis quelque temps déjà nous déplorions l'absence d'une anthologie de la dramaturgie latino-américaine contemporaine en langue française, instrument essentiel pour la diffusion en France et dans la francophonie d'un choix d'œuvres représentatives de cette vaste région du monde. Des pays comme les États-Unis et l'Italie avaient déjà fait l'effort de traduire dans leurs langues respectives une ou plusieurs sélections représentatives d'œuvres latino-américaines.

2 Grâce aux Éditions Unesco paraît aujourd'hui cette anthologie de la dramaturgie latinoaméricaine contemporaine (1940-1990), destinée, en général, à ceux qui s'intéressent à l'Amérique latine dans le monde de la francophonie et, en particulier, au public concerné par le théâtre, aux professionnels et aux amateurs de théâtre, aux professeurs du secondaire, aux universitaires et aux étudiants. 


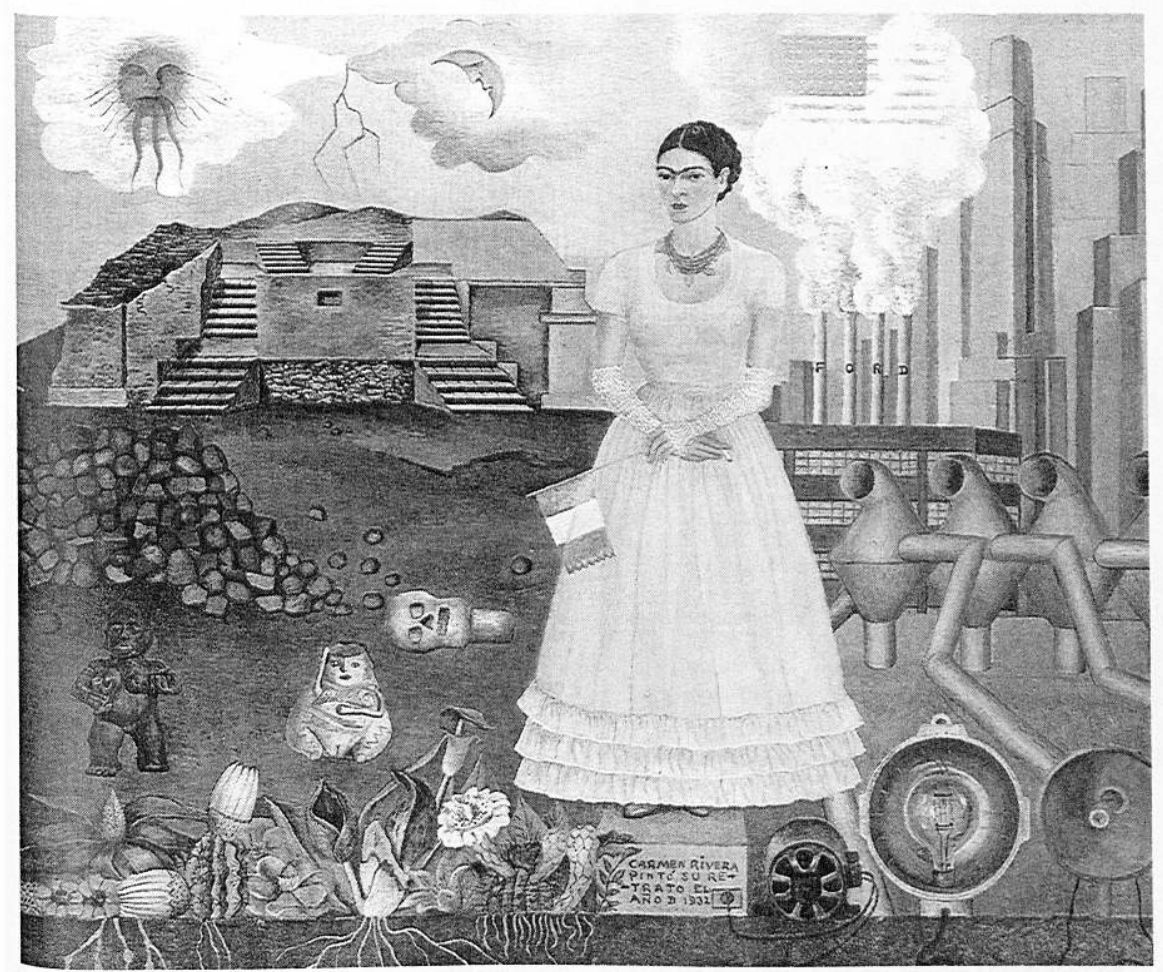

Fondation Pierre Gianadda

3 Avec la complicité de Claude Demarigny (coordinateur de la traduction) nous avons assumé la tâche délicate que nous a confiée Fernando Aínsa, directeur de la Collection d'CEuvres Représentatives de l'UNESCO. Signalons au passage que, sous les auspices de l'Institut de Coopération Intellectuelle de la Société des Nations, fut publié Théâtre choisi, de Florencio Sánchez (1939), choix de cinq œuvres de l'auteur uruguayen. ${ }^{2}$

Le premier dilemme qui s'est posé à nous était de savoir si nous devions nous en tenir, exclusivement ou pas, au théâtre américain de langue espagnole. La plupart des anthologues qui nous ont précédé ont choisi de garder l'unité linguistique, avec l'inconvénient d'exclure le théâtre brésilien, d'une incontestable importance. Nous avons fermement voulu l'intégrer dans cette vaste unité conventionnellement appelée "latino-américaine ", où il occupe une place des plus légitimes. Cela n'aurait pas été possible sans la collaboration d'un spécialiste du théâtre brésilien, le critique Sábato Magaldi, qui a fait le choix de l'œuvre brésilienne.

5 Nous avons dû conjuguer divers facteurs pour déterminer notre sélection. C'est la qualité des textes qui a compté surtout, c'est-à-dire l'ambition du projet, la densité de leur contenu, tout comme leur valeur formelle. Il peut y avoir, indubitablement, des spectacles au succès retentissant, mais bâtis sur un texte sans qualité suffisante pour figurer dans une anthologie de la « dramaturgie».

6 Par ailleurs, nous avons écarté les œuvres brèves, parfois excellentes, pour garder une certaine homogénéité de volume. La grande majorité des anthologies précédentes distinguent ces deux catégories.

7 Autre facteur prépondérant, la sélection d'œuvres « représentatives » de la diversité culturelle de l'Amérique latine en sa vaste géographie. Garder cet équilibre n'a pas été 
chose facile, d'autant que la dramaturgie ne connaît pas le même développement, le même niveau de qualité dans tous les pays et toutes les régions. Le choix préalablement limité à onze pièces aurait déjà rendu impossible la représentation des vingt pays composant le sous-continent, au demeurant inconciliable avec le critère essentiel de qualité. $^{3}$

Il y a des pays de forte tradition théatrale où le choix s'est avéré extrêmement difficile, étant donné le nombre d'auteurs importants. Tel a été le cas pour l'Argentine, qui est représentée par À la campagne (1968) de Griselda Gambaro, la seule femme de notre sélection. Dans cette pièce se condensent avec une particulière intensité quelques constantes du théâtre de G.Gambaro: ambiguïté et déshumanisation du monde représenté, cruauté extrême des rapports entre les personnages, où l'agressivité reste sous-jacente; humour noir et emploi de la parabole pour montrer une réalité qui transcende ce qui est purement local et concret. D'autres auteurs argentins mériteraient aussi d'avoir une place dans cette anthologie, comme Ricardo Monti (1944), Roberto Cossa (1934), Roberto Arlt (1900-1942), Agustín Cuzzani (1924), Carlos Gorostiza (1920), Osvaldo Dragún (1929), Eduardo Pavlovsky (1933)...

9 Même constat pour le Mexique. Photographie sur la plage (1984) d'Emilio Carballido nous semble un texte excellent, qui couronne l'œuvre d'un auteur reconnu internationalement par la qualité de sa production, si vaste et si variée. En présentant une réunion familiale un jour d'été où il ne se passe rien de vraiment exceptionnel, Carballido parvient à une écriture pleine de fines nuances, de laquelle se dégage à certains moments une poésie du quotidien, une certaine atmosphère tchékhovienne. Les personnages apparaissent avec leurs qualités et leurs défauts, et même les antipathiques sont traités avec justesse et indulgence. Mais concernant le théâtre mexicain, il ne faut pas oublier Rodolfo Usigli (1905-1979), Celestino Gorostiza (1904-1967), Xavier Villaurrutia (1904-1950), Elena Garro (1920), Luisa Josefina Hernández (1928), Sergio Magaña (1924-1990), Vicente Leñero (1933), entre autres.

Le théâtre brésilien, qui avait pris du retard par rapport à l'Argentine et au Mexique, a su le combler depuis les années trente. Aujourd'hui la dramaturgie et la scène brésiliennes étonnent par sa créativité et son dynamisme. Chargé du choix de la pièce, Sábato Magaldi a proposé en premier lieu Vestido de Noiva de Nelson Rodrigues, mais sur la liste de sa pré-sélection figuraient aussi Auto da Compadecida d'Ariano Suassuna (1927), Vereda de Salvaçao de Jorge Andrade (1922) et Rasga Coraçao d'Oduvaldo Vianna Filho (1936-1974).

11 Des pays de dimensions plus réduites comme Cuba, Porto Rico, le Chili et l'Uruguay, ont aussi une estimable richesse dramaturgique. La nuit des assassins (1965) de José Triana est sans aucun doute un des meilleurs textes du théâtre cubain, joué partout dans le monde. La réussite de Triana est d'avoir pratiqué une analyse implacable d'une famille de classe moyenne, déchirée par ses conflits et ses frustrations, du point de vue des enfants, en utilisant comme élément structurant la situation du jeu, qui équivaut ainsi à la situation classique du théâtre dans le théatre. La véritable identité des adolescents reste pour cela dans une zone de pénombre, impossible à appréhender vraiment. Mais Cuba possède aussi des auteurs de premier plan, comme Virgilio Piñera (1912-1979), Abelardo Estorino (1925), Rolando Ferrer (1925), Eduardo Manet (1927), devenu un écrivain de langue française, entre autres, sans compter la génération plus jeune très prometteuse. 

l'héritier direct de René Marqués (1919-1979) et Francisco Arriví (1915), pionniers de la rénovation théâtrale dans leurs pays. Ces trois auteurs majeurs ont en commun une même passion pour approfondir la condition portoricaine et l'exigence d'un renouvellement du langage dramatique, appuyés sur une culture et une pratique théâtrales très solides. Cette pièce de Sánchez est profondément marquée par son contexte historique, mais aussi par la tendance, prédominante dans le milieu théâtral « engagé " des années soixante en Amérique latine, à s'identifier pleinement aux principes et procédés brechtiens. L. R. Sánchez actualise le mythe grec en le situant à une époque récente et il l'adapte au monde latino-américain. Si Créon correspond à la figure du dictateur, Antigone a les traits typique d'une métisse américaine. Le même critère est appliqué au reste des personnages, sans tomber à aucun moment dans le «folklore ». Parmi le groupe de jeunes auteurs surgis après Sánchez - plus incliné au roman ces dernières décennies - on voit peser d'un plus grand poids deux figures à plusieurs facettes et au tempérament très différent : José Luis Ramos (1950) et Roberto Ramos Perea (1959).

13 Les envahisseurs (1963) du Chilien Egon Wolff a été représentée partout en Amérique latine. L'admirable dans cette pièce c'est le talent de Wolff pour représenter le contraste entre opulence et misère et sa condamnation explicite de cette situation inhérente à la réalité de l'Amérique latine, mais valable aussi pour le « tiers-monde » et de plus en plus pour les pays hautement industrialisés, avec ses légions de chômeurs, ces nouveaux parias du système. Jusqu'à présent et autant qu'on le sache, le traitement du sujet par Wolff reste inégalé dans le cadre de l'Amérique latine, enrichi et approfondi, de surcroît, par ses deux pièces postérieures : Flores de papel (Fleurs de papier, 1970) et La balsa de la Medusa (Le radeau de la Méduse, 1984)4, ce qui justifie l'accueil reçu par ces pièces et la place méritée qu'elles occupent dans les anthologies nationales et continentales. Aux côtés de Wolff, Jorge Díaz (1930), Luis Alberto Heiremans (1928-1964), Sergio Vodanovic (1926) et Isidora Aguirre (1919) occupent une place importante, suivis de quelques auteurs plus jeunes comme Juan Radrigán (1937) et Marco Antonio de la Parra (1952).

(1977, version définitive) de l'Uruguayen Ricardo Prieto, est une pièce qui surprend dans la mesure où les situations quotidiennes se transforment imperceptiblement en cauchemar, en un monde hallucinant, insolite et suggestif. Le recours utilisé consiste à provoquer une situation apparemment banale et à la pousser à l'extrême, voire au paroxysme, avec une cohérence propre qui n'est pas précisément rationnelle. Nous sommes très loin $\mathrm{du}$ théâtre naturaliste de Florencio Sánchez (1875-1910), pionnier de la dramaturgie de ce pays. Entre Sánchez et Prieto, il faut mentionner Carlos Maggi (1922-), Jacobo Langsner (1927-), Mauricio Rosencof (1933-) et Carlos Manuel Varela (1940-), parmi les plus connus.

15 La Colombie et le Venezuela ont une tradition théâtrale plus récente, mais de grande vitalité ces derniers temps. Les vieilles malles poussiéreuses que nos parents nous ont interdit d'ouvrir (1968) du Colombien Carlos José Reyes nous semble très représentative de la société colombienne contemporaine. Cette pièce n'a pas perdu de sa force ni comme portrait satirique d'une famille aisée, avec ses préjugés, ses secrets et ses mensonges, ni comme perception de la société colombienne et, par extension, latino-américaine, fondée sur l'égoïsme et la violence. Nous sommes très conscients de l'apport d'Enrique Buenaventura (1925) au théâtre colombien depuis les années soixante, en tant 
qu'auteur, metteur en scène et maître des nouvelles générations, mais au moment de choisir le meilleur texte nous avons préféré Les vieilles malles...

Rêverie d'un dimanche après-midi au parc Alameda, Diego Rivera, 1947

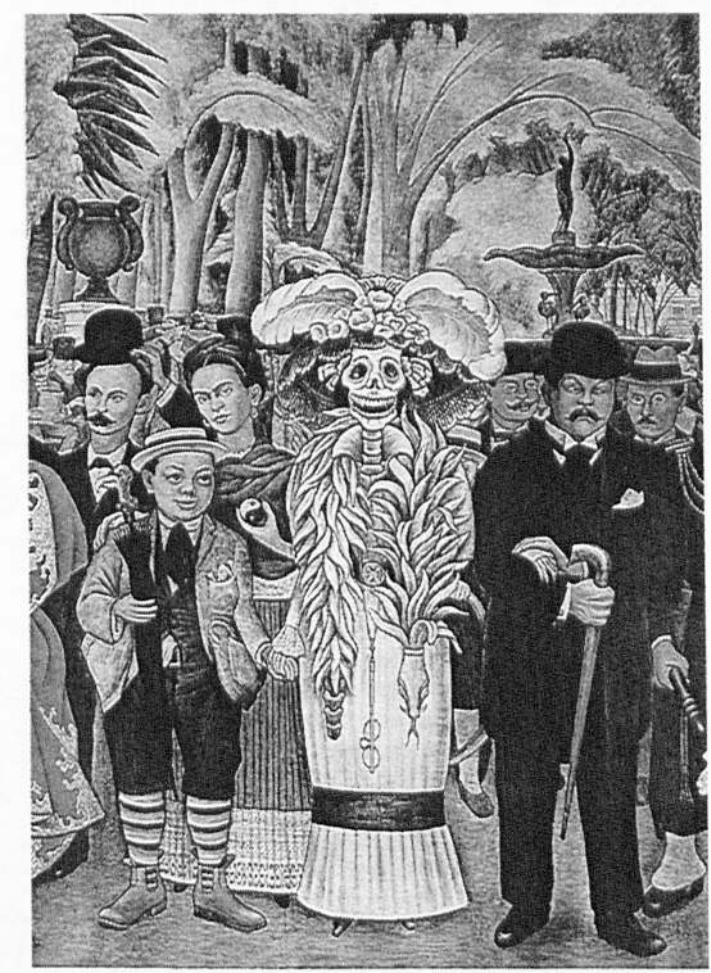

Fondation Pierre Gianadda

Acte culturel (1975) de José Ignacio Cabrujas n'a pas cessé de confirmer sa place de premier plan dans la dramaturgie vénézuélienne, C'est une œuvre de maturité à l'intérieur de l'évolution de l'auteur, au même titre que El día que me quieras (Le jour où tu m'aimeras, 1979). Le choix de l'«acte culturel» comme support de base de l'action nous semble déterminant, non seulement quant à sa structure, mais aussi quant aux personnages, aux rapports entre et avec le public virtuel auquel fait allusion le texte et, en dernier lieu, à la recherche sur l'identité culturelle vénézuélienne. De la même génération et d'un niveau semblable de qualité en tant que dramaturges, ses complices de Nouveau Groupe: Isaac Chocrón (1932) et Roman Chalbaud (1931). Plus jeune, Rodolfo Santana (1944) se profile comme leur successeur indiscutable.

Pour leur part, Soluna (1955) de Miguel Angel Asturias (Guatemala) et Collacocha (1956) d'Enrique Solari Swayne (Pérou), représentent aussi, d'une certaine manière, la dramaturgie de l'Amérique centrale (Nicaragua, Honduras, El Salvador, Costa Rica et Panama) et celle des pays andins (L'Équateur et la Bolivie), respectivement. Soluna est une pièce métisse par excellence, un mélange réussi de la culture européenne et de la culture maya-quiché. Le sous-titre: "Comédie prodigieuse» annonce déjà la prédominance des sources mythologiques et de croyances chamaniques, tellement enracinées encore parmi les Indiens et les paysans d'Amérique centrale. Asturias montre - à partir d'un épisode amoureux - les différents niveaux de réalité et la complexité de la condition humaine, que les canons rationalistes en eux-mêmes sont parfois insuffisants pour l'interpréter. Asturias a jeté les bases d'un théâtre original 
guatémaltèque, qui a été enrichi ultérieurement, surtout par Manuel Galich (1913-1984) et Manuel José Arce (1936-1985).

Le sujet principal de Collacocha est le combat titanesque de l'homme pour dominer la nature, sujet traité souvent par le roman latino-américain des années 20, mais rarement par le théâtre, pour des raisons évidentes. Plus que de style réaliste, à propos de Collacocha, il faudrait parler d'hyperréalisme, au sens d'une intensification du réalisme avec l'amplification de ses composantes, comme la démesure du personnage central (l'ingénieur Echecopar) et le combat, qui relève du mythe de Sisyphe, pour construire un chemin dans le ventre des Andes majestueux, ainsi que l'ampleur du mouvement sismique qui détruit une œuvre presque achevée. L'ambition du projet de Solari Swayne et sa concrétisation scénique imposent le respect, même si on ne croit pas dans la panacée du progrès technique, qui peut être aussi nocif, voire destructeur (Hiroshima et Nagasaki le prouvent), si l'on oublie l'essentiel : l'homme. À part Solari Swayne, la dramaturgie péruvienne est représentée par Sebastián Salazar Bondy (1924-1965), probablement le plus important de tous les auteurs dramatiques pour l'ensemble de son œuvre, Juan Rivera Saavedra (1930), Alonso Alegría (1941), entre autres.

La lecture totale de ces œuvres devrait fournir au lecteur une idée générale de l'évolution de la dramaturgie latino-américaine sur le plan des tendances esthétiques qui ont jalonné ou marqué la seconde moitié du $\mathrm{XX}^{\mathrm{e}}$ siècle.

\section{NOTES}

1. Cet article est un fragment de l'introduction de l'ouvrage.

2. Florencio Sánchez, Théâtre choisi, Paris, Institut international de coopération intellectuelle, 1939 (Traduction de Max Daireaux et préface d'Enrique Diez Canedo).

3. Ma proposition initiale comportait douze pièces, dont deux argentines, étant donné le nombre de dramaturges argentins de grande qualité. Pour des raisons qui relèvent strictement de critères d'édition (surtout le nombre de pages), nous avons gardé À la campagne de Griselda Gambaro au lieu d'Une passion sud-américaine de Ricardo Monti, un texte de valeur également indiscutable.

4. Pièce publiée dans Coulisses $n^{\circ} 16$, mai 1997, dans la traduction de Carole Michel. Voir également le dossier consacré au théâtre d'Egon Wolff sous ma direction dans Coulisses $n^{\circ} 16$ et 17. 